$\cdots$

Atomics International Division

Rockwell International
U

CLASSIFICATION LEVEL

(S, COR U)
DOCUMENT NO

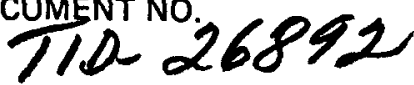

TR -653-240-004

\title{
COVER SHEET
}

DOCUMENT TITLE

Machining Capability of Hobbing SNAP Cladding Fins
AUTHOR

F. C. Schrag
Thu report was prepared as an account of work sponsored by wh prepared as an account of work the Unted States nor the United States Energy Research and Development Admunstration, nor any of thets employees, nor any of then contractors, subcontractors, or ther employees, makes any labulty or responsubuty for the or assumes any lega or usefulnes of any information appas, was, pleteness process discloved, or represents that its use would on infringe privately owned rughts

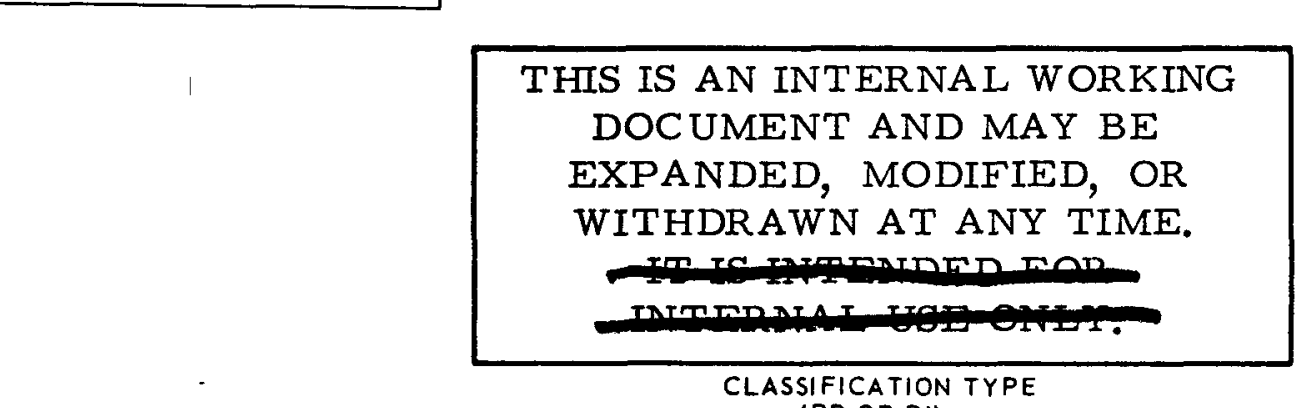

(RD OR DI)

NOTICB

PORTICNS OF THIS REPORT ARE IILEGTBLF. W cony to r. rait tho br in the best availablo abiliti.

\section{THIS IS AN INTERNAL WORKING \\ DOCUMENT AND MAY BE EXPANDED, MODIFIED, OR WITHDRAWN AT ANY TIME.}

CLASSIFICATION TYPE

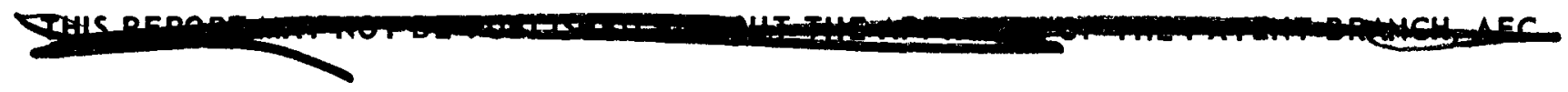

This report was prepared as an account of work sponsored by the United States Government Neither the United States nor the United States Atomic Energy Commission, nor any of their employees nor any of their contractors, subcontractors, or their employees, makes any warranty, express or implied, or assumes any legal liability or responsibility for the accuracy, completeness or usefulness of any information, apparatus, product or process disclosed, or represents that its use would not infringe privately owned rights 


\section{DISCLAIMER}

This report was prepared as an account of work sponsored by an agency of the United States Government. Neither the United States Government nor any agency Thereof, nor any of their employees, makes any warranty, express or implied, or assumes any legal liability or responsibility for the accuracy, completeness, or usefulness of any information, apparatus, product, or process disclosed, or represents that its use would not infringe privately owned rights. Reference herein to any specific commercial product, process, or service by trade name, trademark, manufacturer, or otherwise does not necessarily constitute or imply its endorsement, recommendation, or favoring by the United States Government or any agency thereof. The views and opinions of authors expressed herein do not necessarily state or reflect those of the United States Government or any agency thereof. 


\section{DISCLAIMER}

Portions of this document may be illegible in electronic image products. Images are produced from the best available original document. 


\section{Atomics International North American Pockwell}

No. . IR-653-240-a)

PAGE. 2

\section{CBJACTIVE}

The objective of this test was to establish the machining capability of bobbing fins on SKuP cladding.

\section{DISCOSSION}

In order to froride coolant wixing within the sMP 8 reactor core, it is desirable to have helical fins achized on some of the fuel elements. (i) An example of a finned element wth a thermocouple attached is shown in Figure 1. The detailed drawing of a linned cladding tube is shown in Figure 2. The technique used to achine helical fins is hobbing. (2)

Fifty Incoloy 800 tubes approximately 21 inches long were wachined by hobbing. The machining was performed by Gear Supply \& Broaching Co., authorized by P.0. FN2150070. The 6tarting material was .540 inch ID by .050 inch wall thickness. The tubing was procured on P.0. W700803 and was within the requirements of STO160NB0037, "Ni, Fe, Cr Alloy Wrought Products." Data for the starting tubing are published in Reference 3.

\section{PROCEDURE}

Each tube was placed on a mandrel and placed between centers of the hobbing machine as shown in Figure 3. A tube support bushing, mounted to the hob catter carriage, supported the tube. This tooling arrangement reduced tool chatter and assured a good surface finish.

The ends of the tubes without fins were machined on a grinding machine. Machining the tube ends on this second machine produced an undercut at the 


\section{Atomics International North American Rockwell}

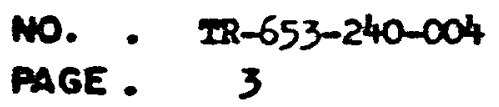

end of the fins. The undercut was exaggerated by a lack of concentricity between the ends and the finned section.

Dimensions of the tubes were recorded after hobbing. Copies of the date sheets are ettached. The wall thickness was measured with a Vidigage. Four thickness readings were recorded for each of 5 positions along the length of the tubes. Tube length, fin length, helical pitch and fin widh were measured with micrometers and calipers. The radius at the root of the $f$ in and fin end vere measured with a radius gage, and undercut at the end of the fins was . measured with an optic comparator. The inside diameter was measured with a trimicrometer. Surface finish was measured with a profizometer and rerfied with visual standards of milling machine finishes.

\section{SUMAMRI OF DATA}

A summary of the dimensional data is presenied in Table 1. Dimensional averages and corrected standard deviations are presented. Results of a comparison of the dimensions with tre drawing requirements are tabulated in Table 2. The dimensions which do not meet the print requirements are the finned length, unfinned length, helical pitch, and wall thickness. A comparison of 3 sigma dimension values, with respect to the drawing tolerances, show the fin length sigma value is the only one which exceeds the specified tolerance requirement.

\section{v. ATTACHMENTS}

Attached to this report are copies of:

1. Purchase order for hobbing.

2. Receiving inspection data.

3. Inspection Internal Discrepancy Report (IDR).

4. Trip Report to Gear Supply Co. 


\section{Atomics International North American Rockwell \\ No. - $2 R-653-240-004$ \\ PAGE: 4}

II. Conculosions

The hobbing process will generate acceptable helical fins on the outside of shap 8 fuel elewents. The three sigan ralues for the dimensions are within the tolerance requirements shown on the drawing, Fig. 3. Some nominal dimensions were grossly outside the nominal requirewents; therefore, the product was rejectable. Improvewent in machine setup will eliminate these rejected conditions. Assurance of proper machine setup could be achieved by inspecting the first fex parts machined at the vendor's plant.

VII. REFERDENCES

1. II, E. Moody to E.J. Donovan, "Scalloped Fins," 3-19-71

2. Lawrence E. Doyle, "Manufacturing Processes and Materials for Engineers"

3. T.D. Williams, "Receiving Inspection Incoloy 800 Fuel Cladding," 3-22-71 


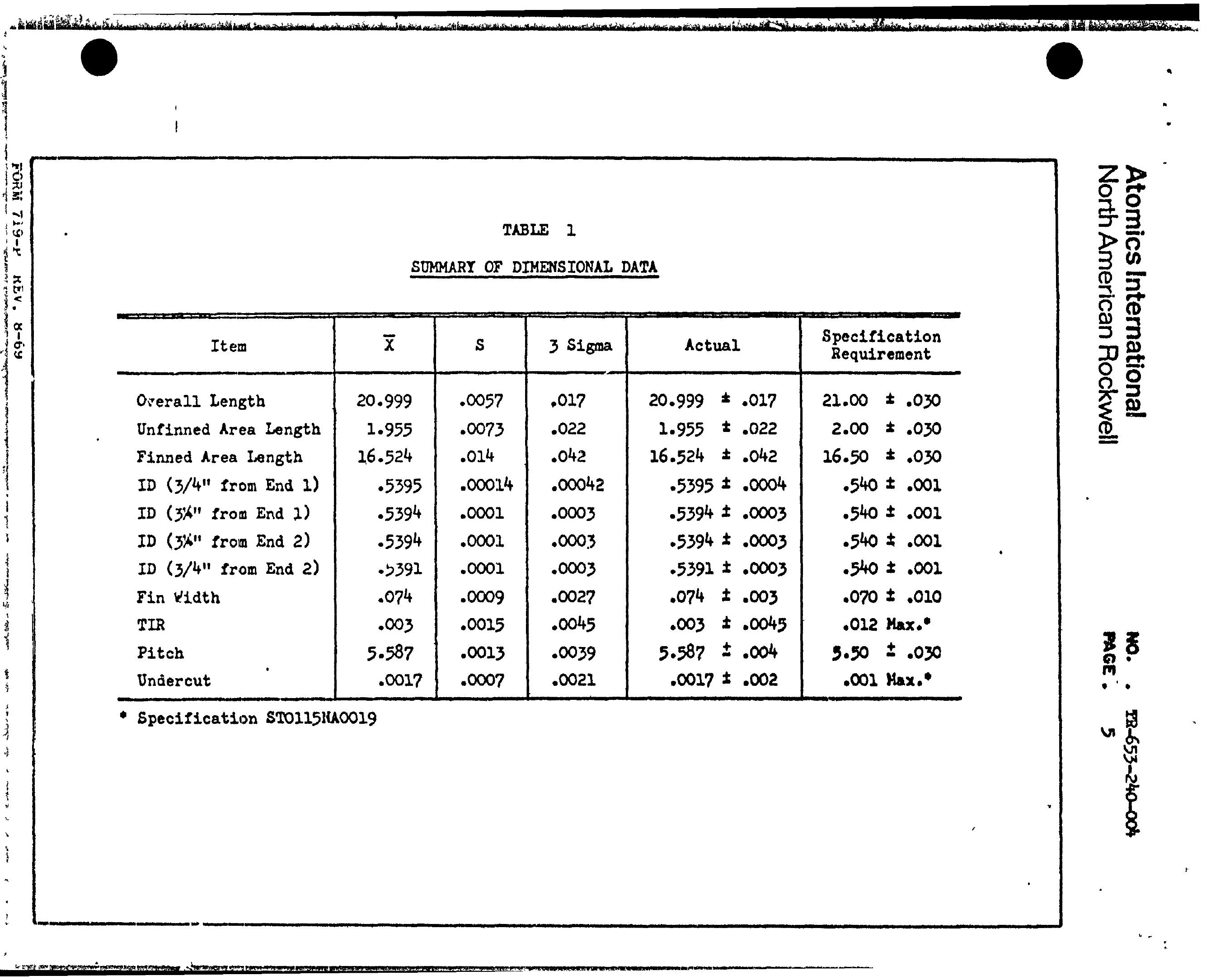




\section{TABLE 2 \\ COMPARISON OF DTAENSIONS \\ TO SPECIFICATION BDOUIRDYENTS:}

\begin{tabular}{|c|c|c|}
\hline Iten & $\begin{array}{l}\text { Kumber } \\
\text { Accepted }\end{array}$ & $\begin{array}{l}\text { Number } \\
\text { Rejected }\end{array}$ \\
\hline Overall Iength & 10 & 0 \\
\hline Unfinned Area Length & $\mathbf{0}$ & 20 \\
\hline Finned Area Length & 8 & 2 \\
\hline Pitch Iength & 0 & 10 \\
\hline End of Fin Radius & 10 & 0 \\
\hline $\mathbf{W D}$ & 10 & 0 \\
\hline F-n Hath & 10 & 0 \\
\hline Fin Radius (Fillet) & 10 & 0 \\
\hline Hall Thickness & 0 & $\cdot 10$ \\
\hline TIR & 10 & 0 \\
\hline Undercut & 10 & 0 \\
\hline
\end{tabular}




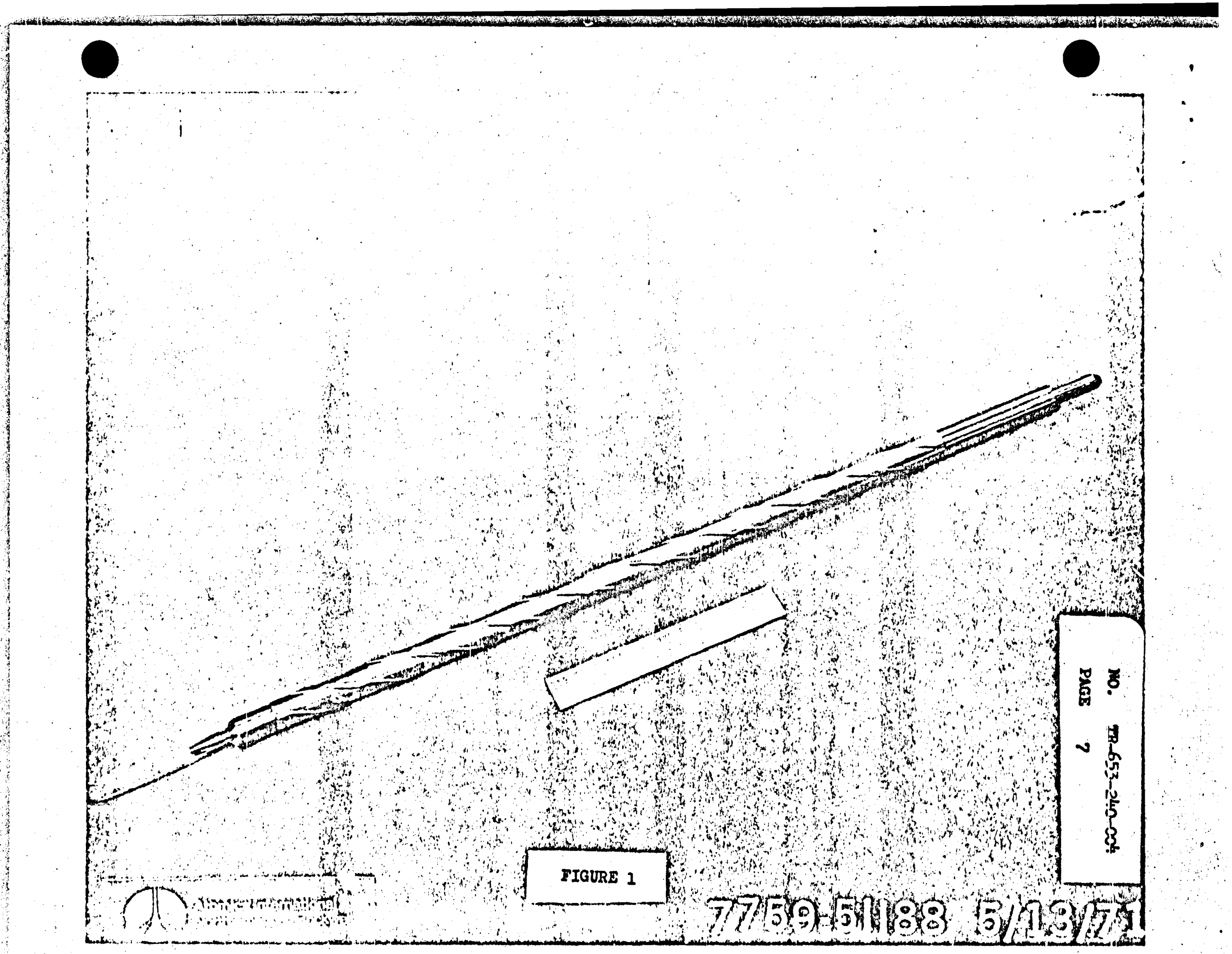




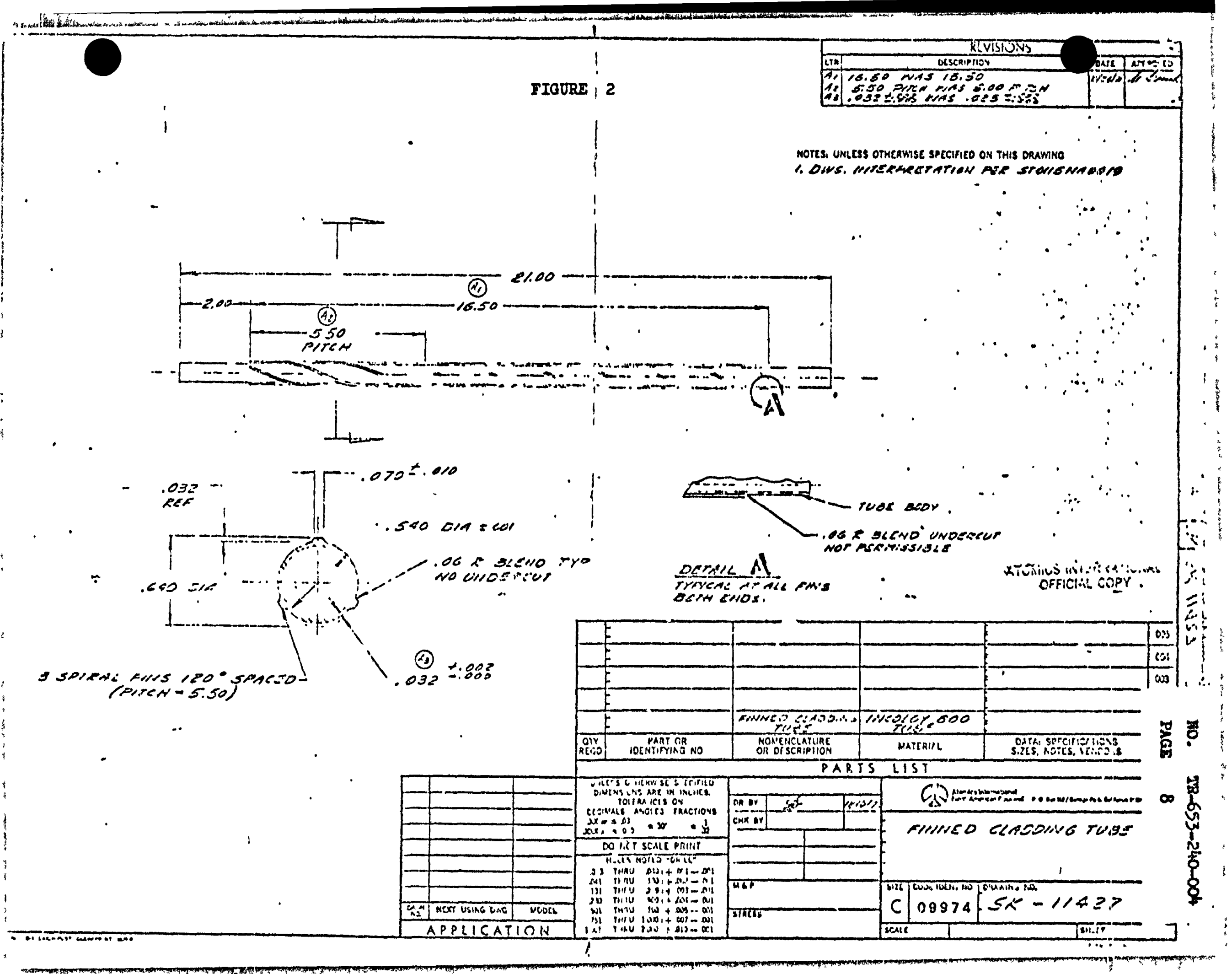




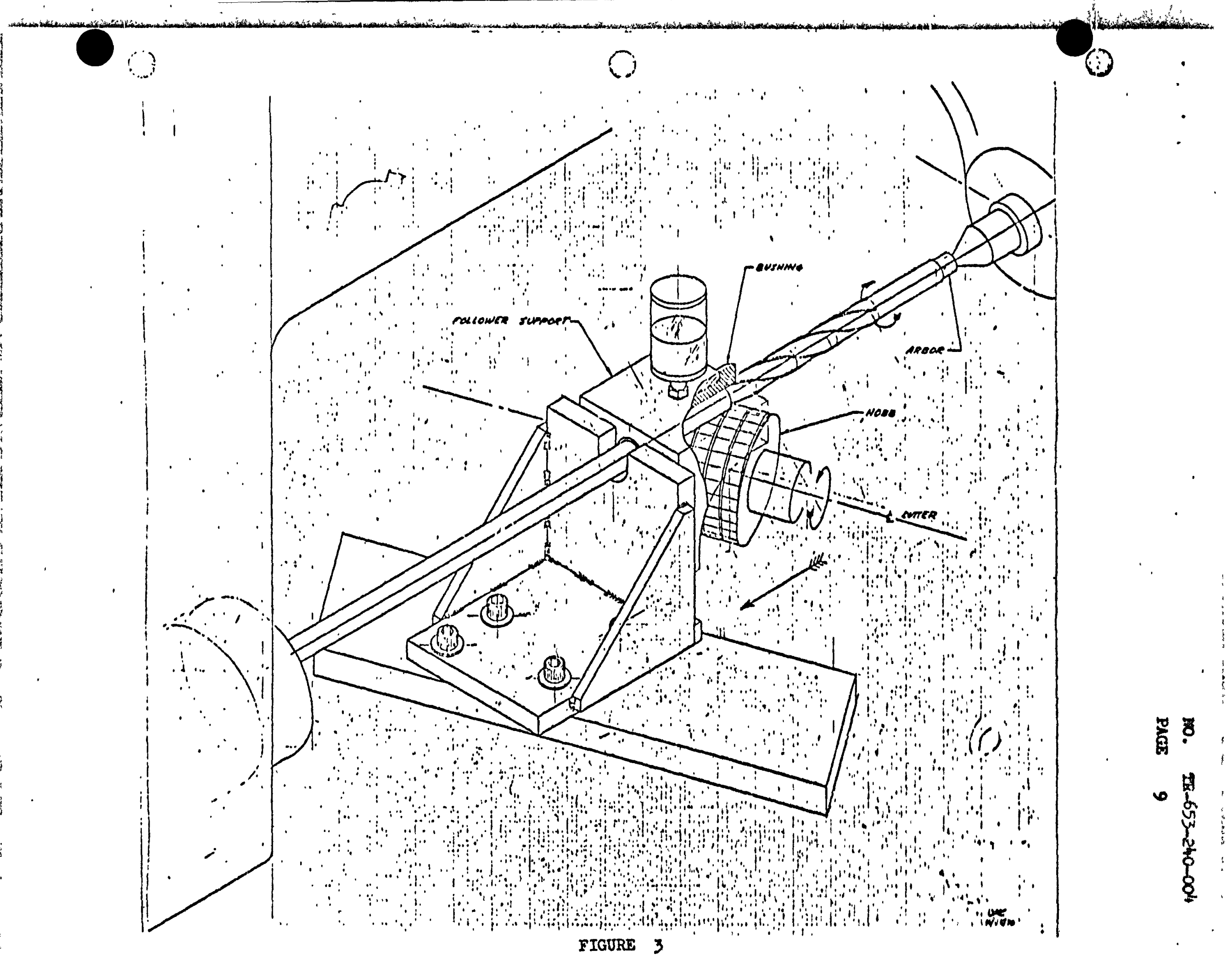


Dents now

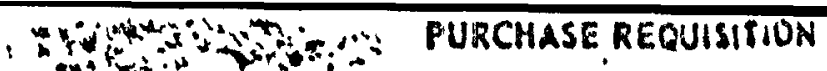

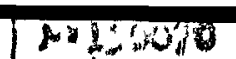

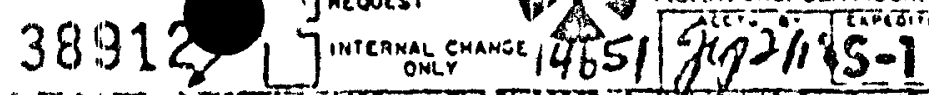

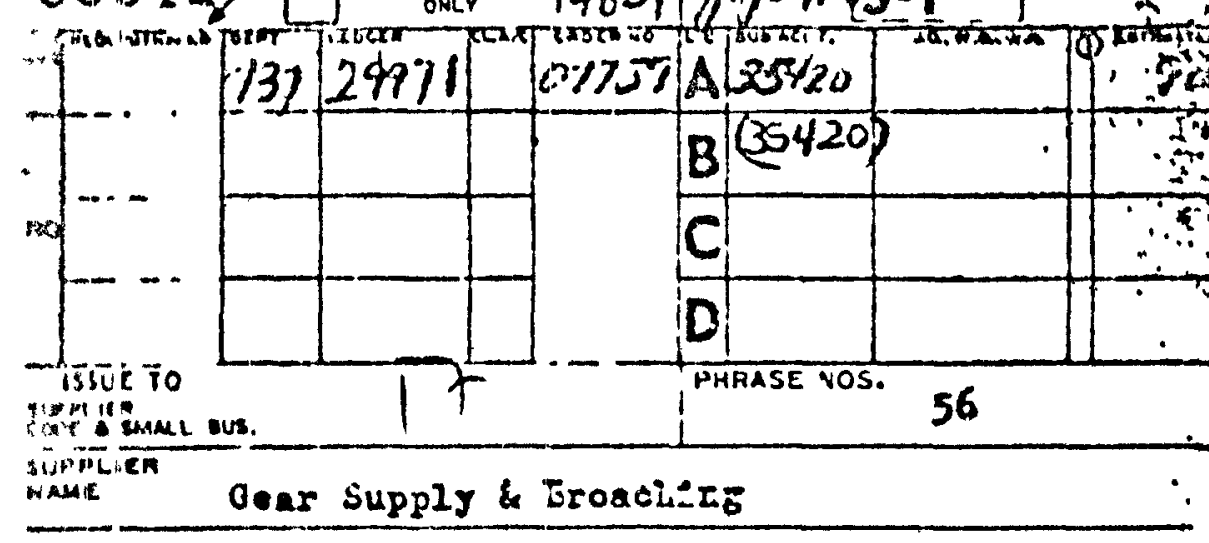
r.t.

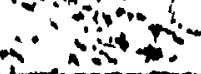

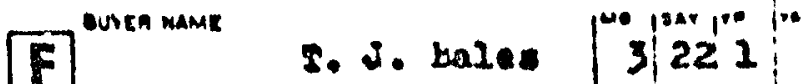

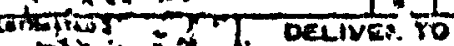

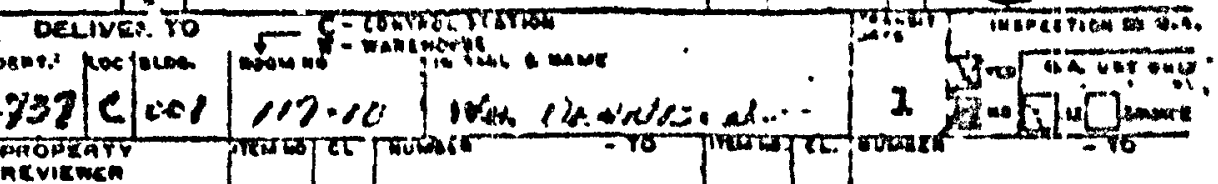
ris $732 \mid c$

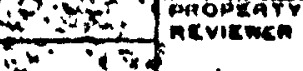

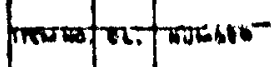

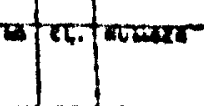

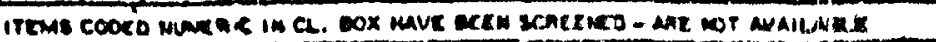
$\because \ldots$ SHIP TOI

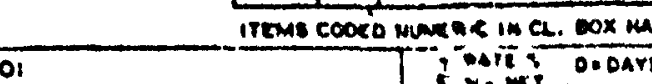

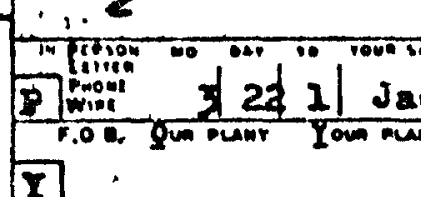

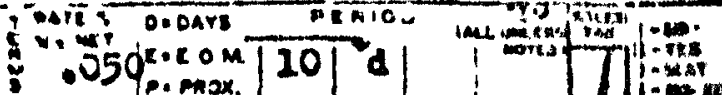

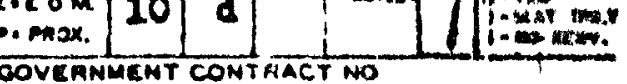

\begin{tabular}{|c|c|c|}
\hline 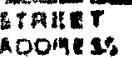 & 10422 5tanford drenue & \\
\hline STAYG & Garden Grove, Califorala & 92640 \\
\hline
\end{tabular}

SHïnivia sumiace Án

s Bujor's Truck

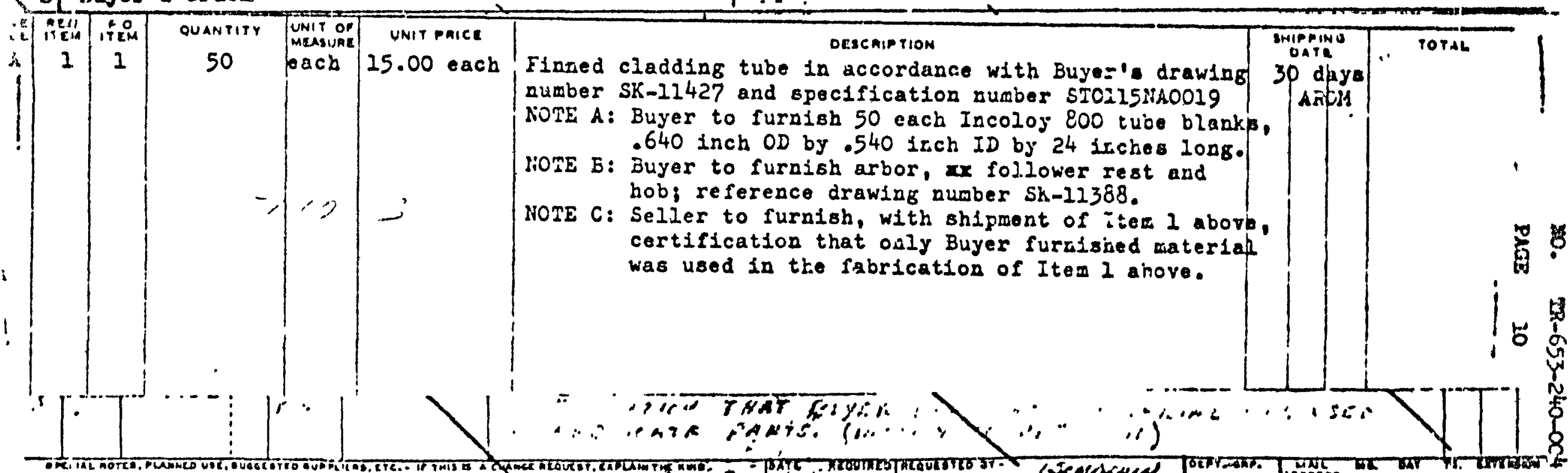
or

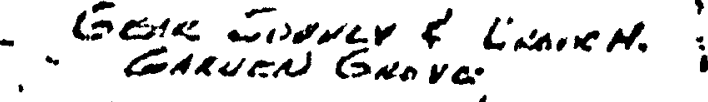

GRow GCAR

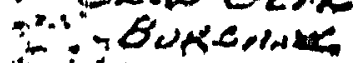

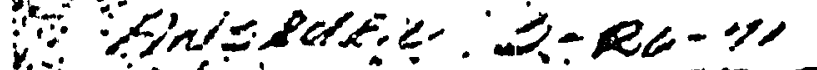

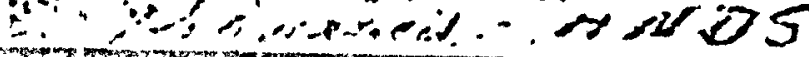

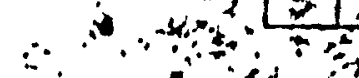

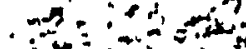

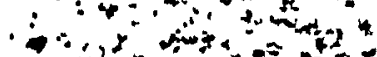

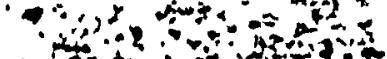
0130 . $\therefore 2^{2} y^{\circ}$ $\therefore$ if EunOs

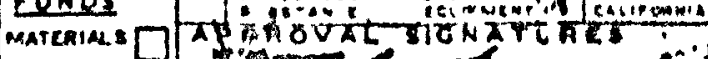

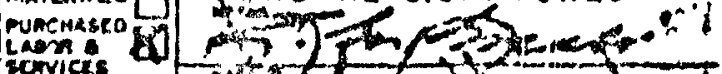
comicis a)

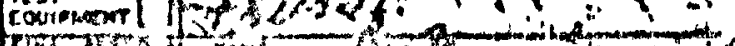
maxprat at 4. 


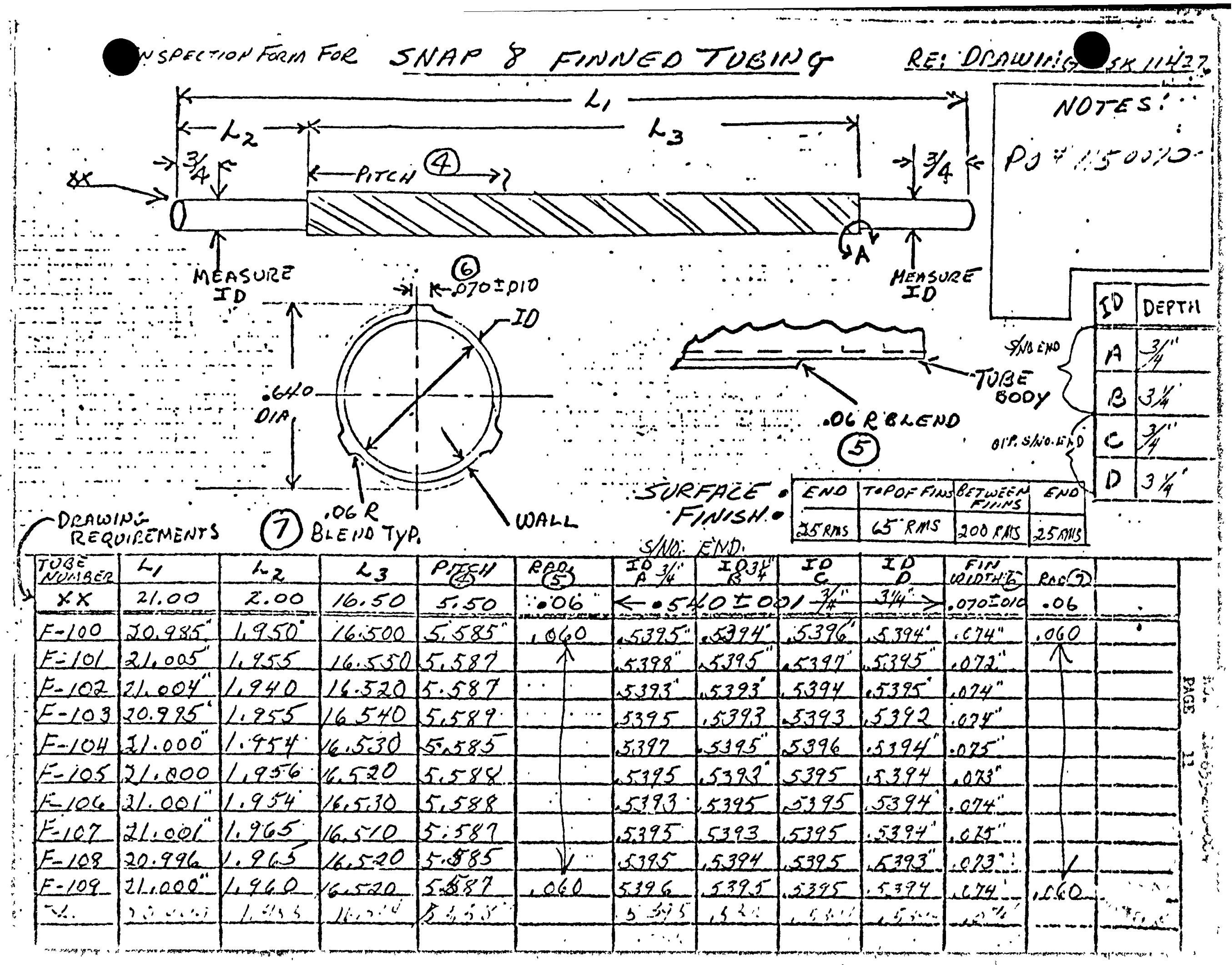




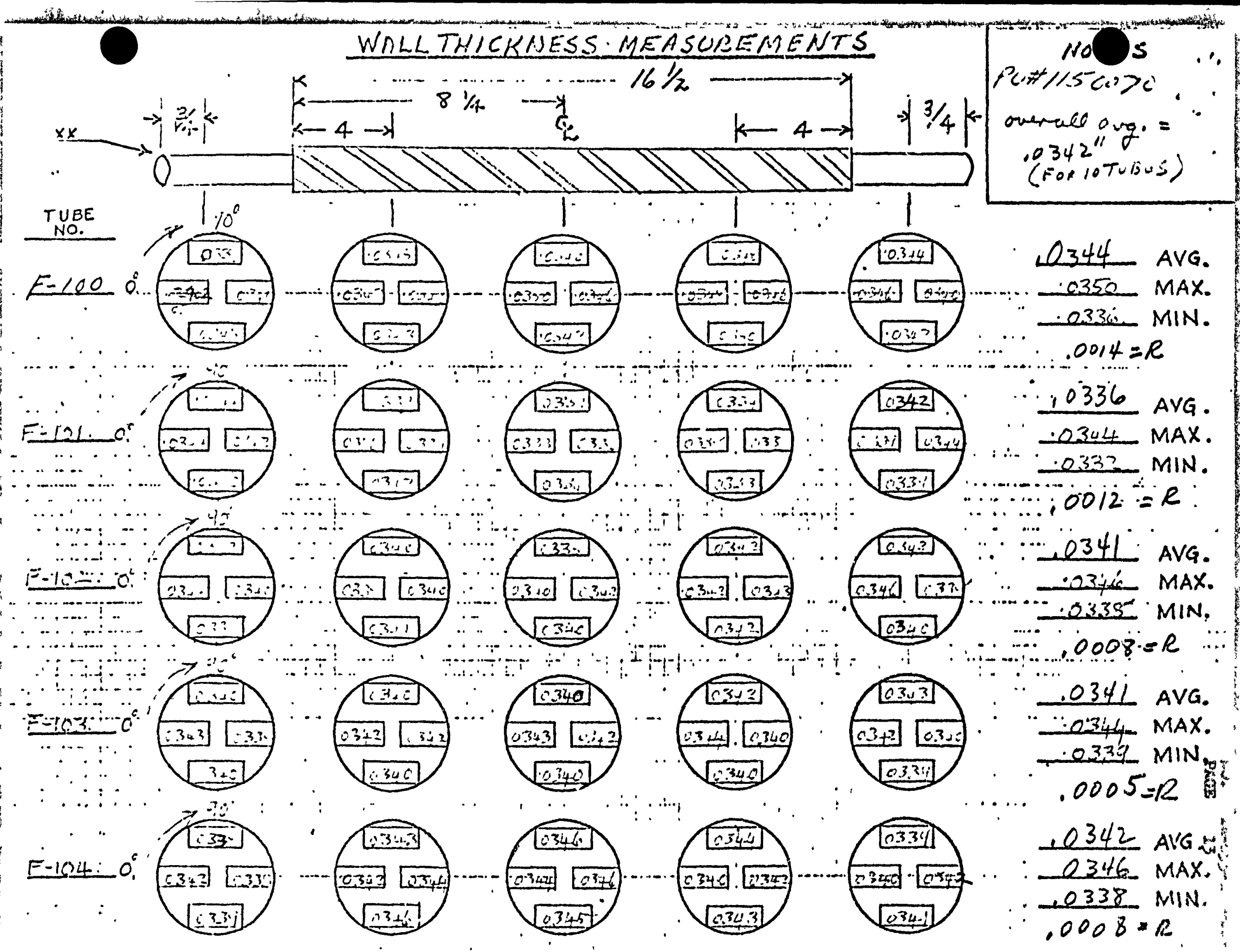


Atomics International

North American Rockwell м0. - T2-653-240-004

PAGE. 14

P.0. $\# 1150070$

WALI THICKNESS

SUAP 8 TUBES

\begin{tabular}{|c|c|c|c|c|c|}
\hline \multirow{2}{*}{$\begin{array}{l}\text { Mube } \\
\text { No. }\end{array}$} & \multirow{2}{*}{$\begin{array}{c}\text { End } \\
\bar{X}\end{array}$} & \multicolumn{3}{|c|}{ Hobbed Section } & \multirow{2}{*}{$\begin{array}{c}\text { Find } \\
\overline{\mathbf{x}}\end{array}$} \\
\hline & & $\overline{\mathbf{x}}$ & $\overline{\mathbf{x}}$ & $\overline{\mathbf{x}}$ & \\
\hline 100 & .0341 & .0343 & .0346 & .0348 & .0343 \\
\hline 101 & .0342 & .0336 & .0334 & .0335 & .0341 \\
\hline 102 & .0342 & .0340 & .0340 & .0342 & .0342 \\
\hline 103 & .0340 & .0341 & .0341 & .0342 & .0341 \\
\hline 104 & .0339 & .0344 & .0345 & .0342 & .0341 \\
\hline 105 & .0341 & .0342 & .0341 & .0342 & .0343 \\
\hline 106 & .0345 & .0344 & .0343 & .0343 & .0343 \\
\hline 107 & .0343 & .0342 & .0344 & .0344 & .0342 \\
\hline 103 & .0343 & .0347 & .0345 & .0345 & .0340 \\
\hline 109 & .0340 & .0343 & .0344 & .0344 & .0342 \\
\hline Avg. $(\bar{X})$ & .0342 & .0342 & .0342 & .0343 & .0342 \\
\hline
\end{tabular}




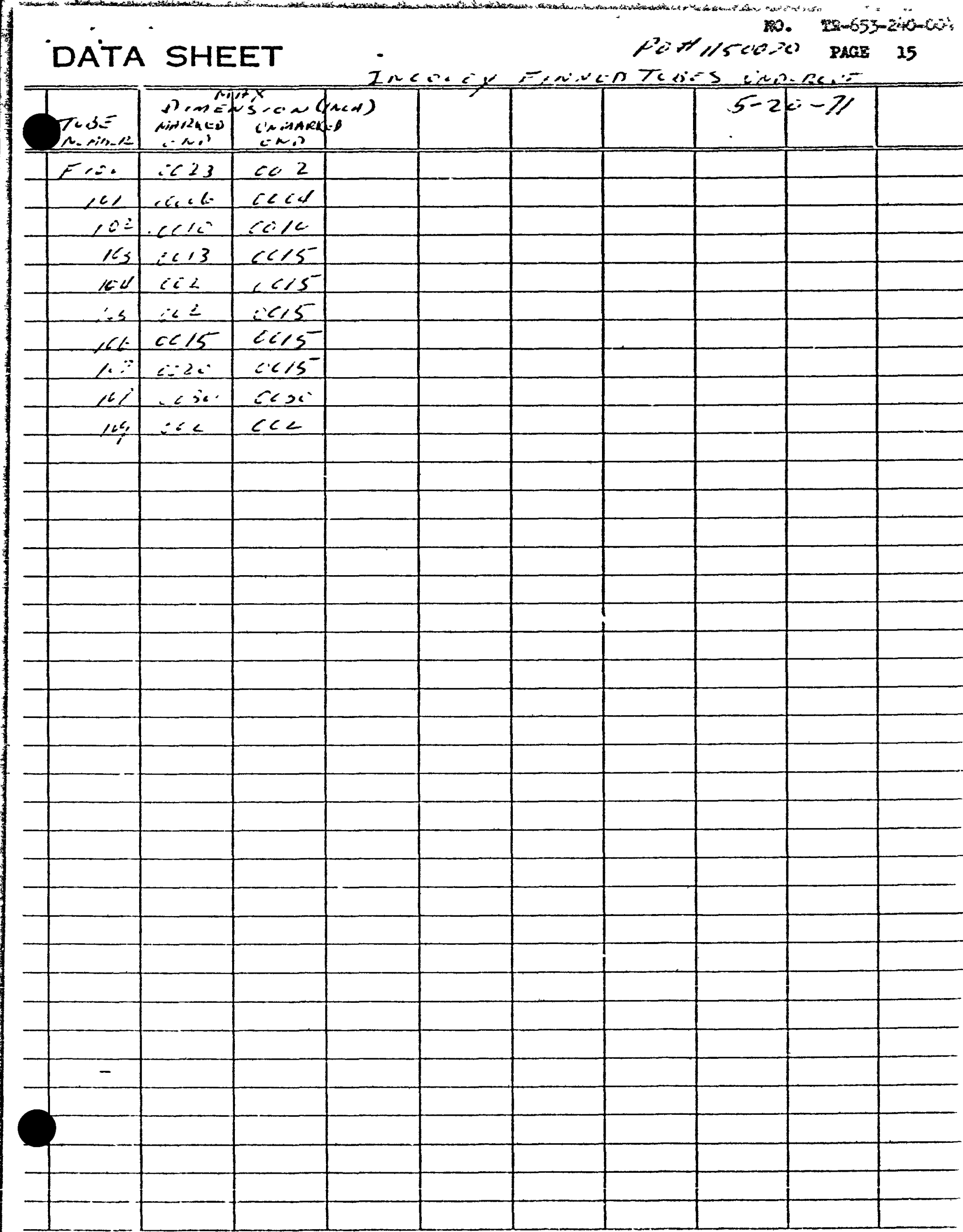




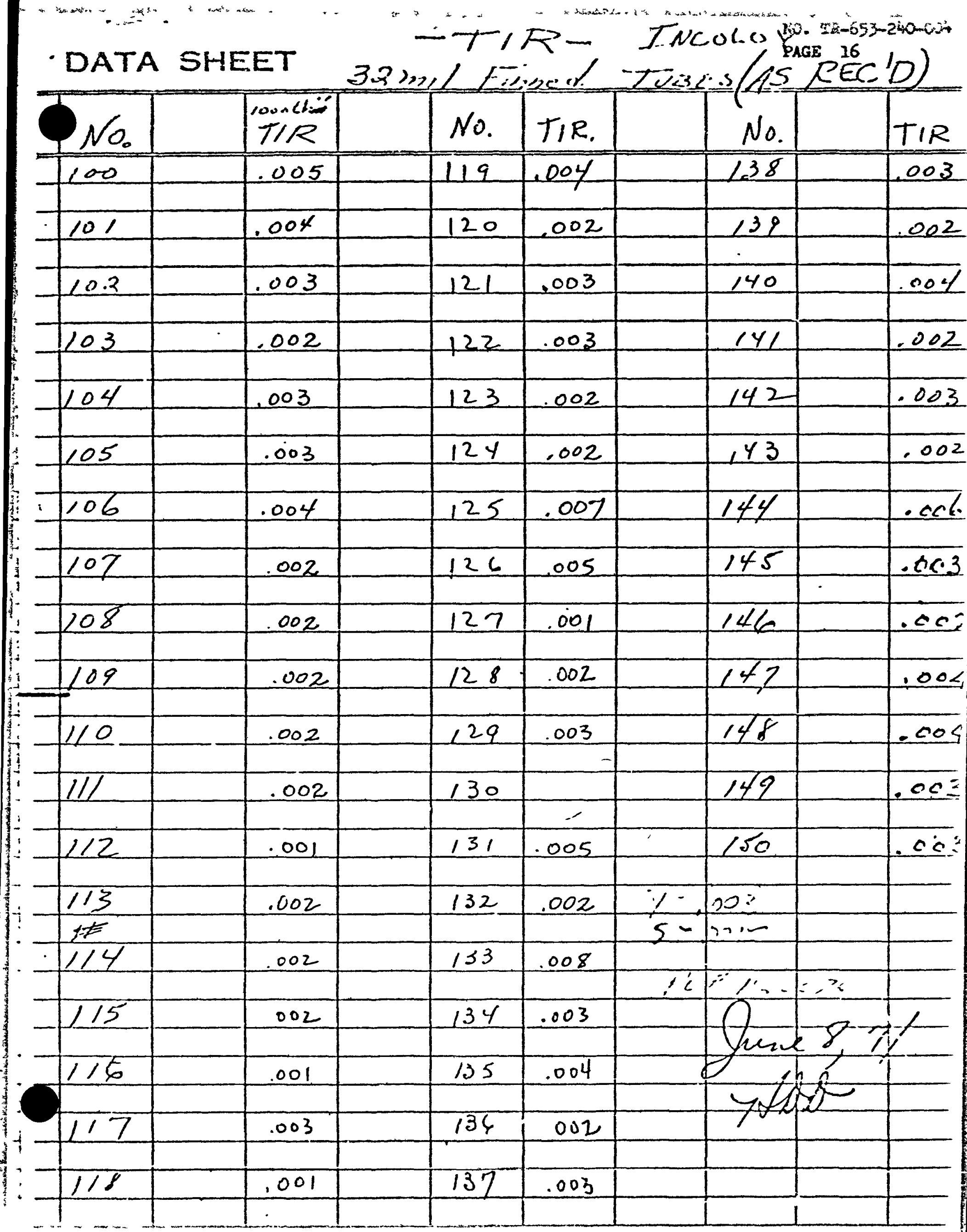




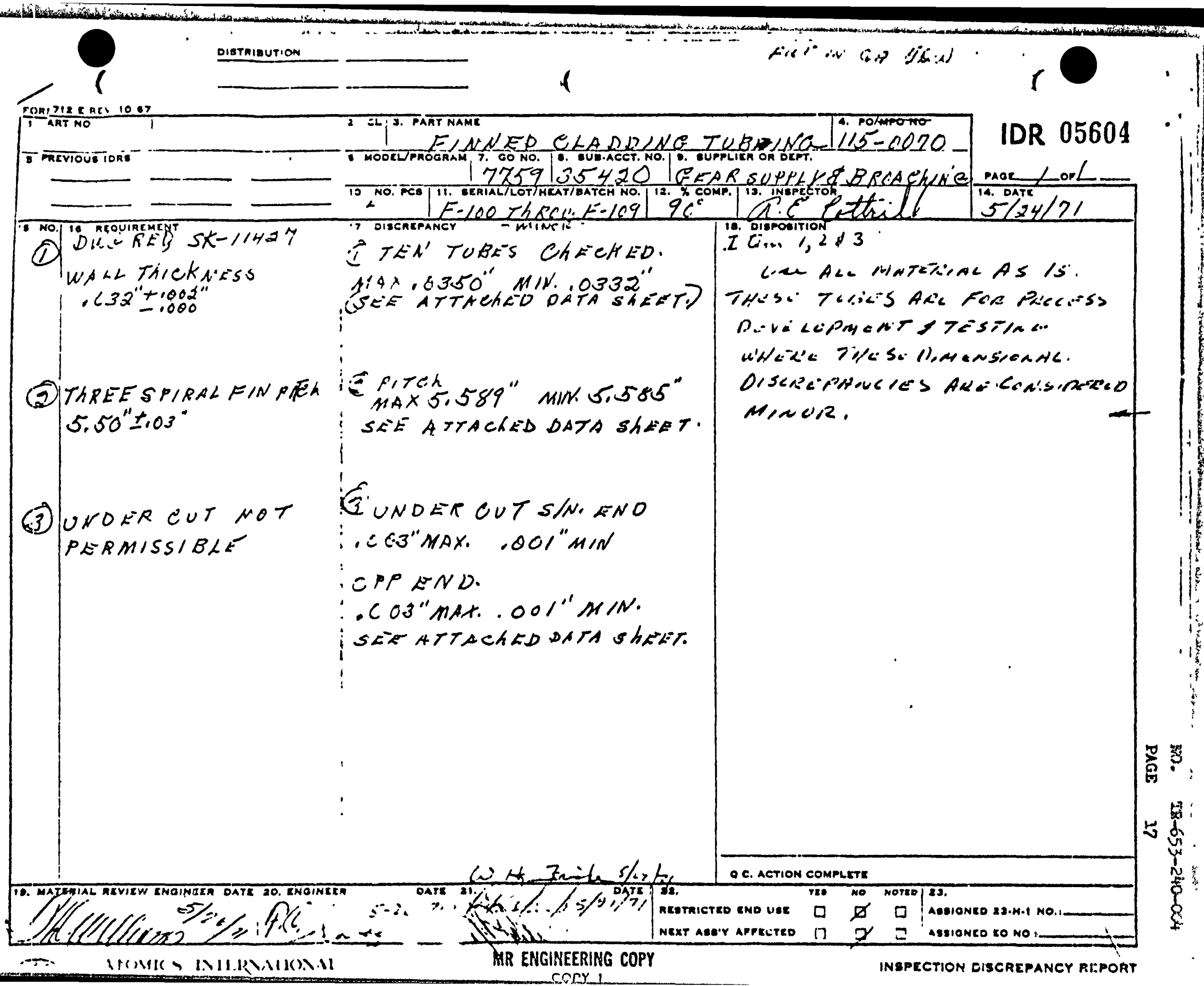




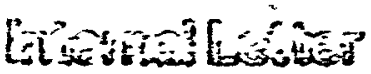

cis

ET

$2 \pi+3$
Axril 30, 1971

D.c. Canjpbell

737-72 1345

No. TR-653-240-004
$\therefore-18$

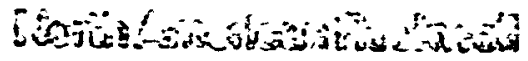

i\%

Bin: $\sqrt{\text { R.c. Scluraz }}$

As:-x: $737-72$ It:45

Fat:e $\quad 1313$

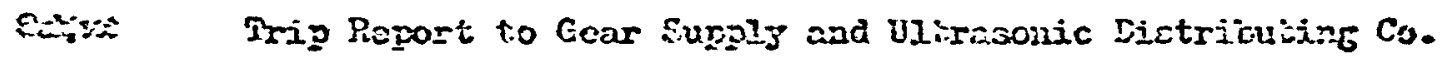

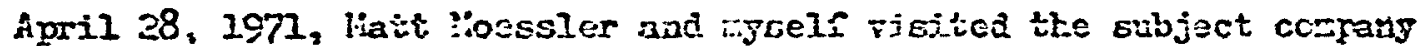

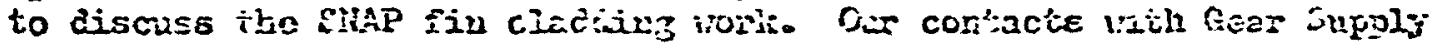
vere Jack Sariore anà foj Jenisins.

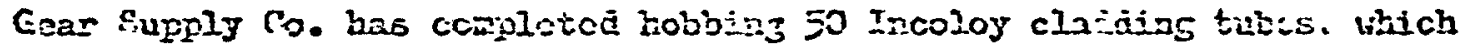
ve bere able to cxamize. The surface firish for these tubes aprears

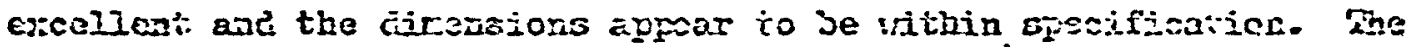

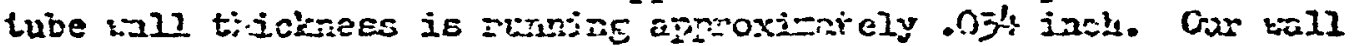
thicierase stecification regiresent is .052 +.002. - .000 inch. Ue

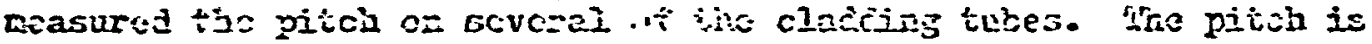

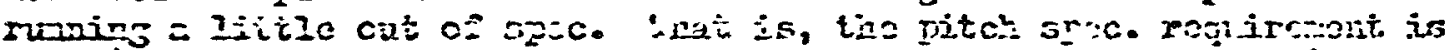

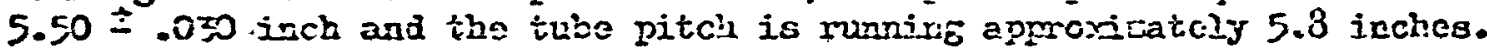

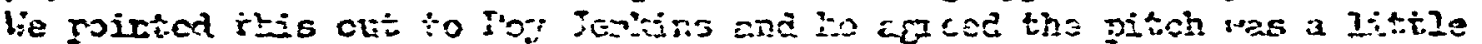

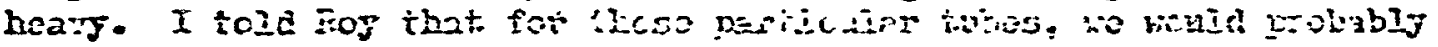

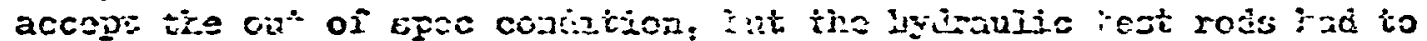

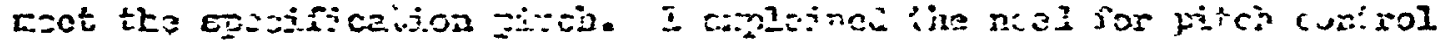

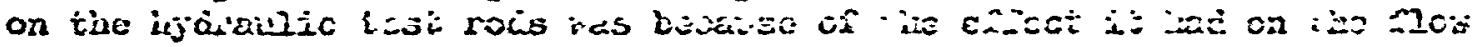
characteristics.

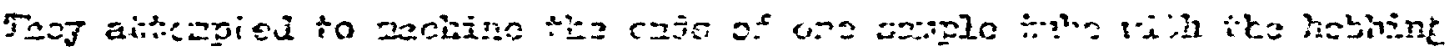

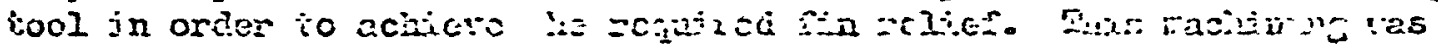

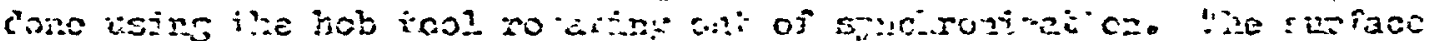

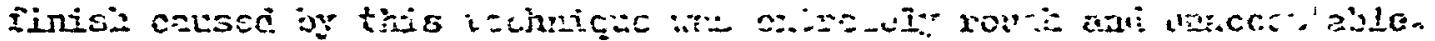

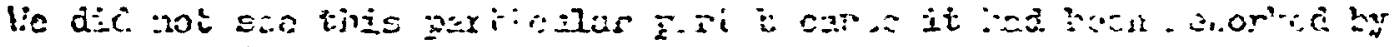

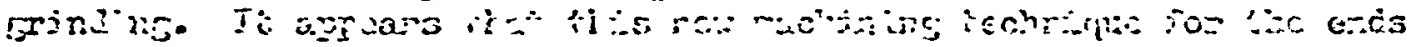
is unacestabio.

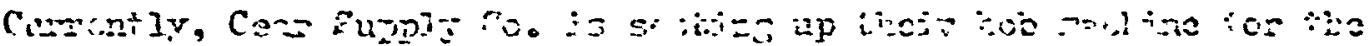

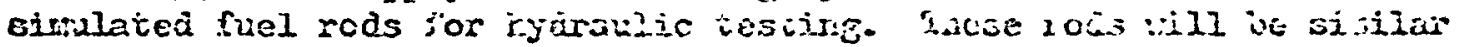
in configuration and jianctcs to ite eost recent cosign of the jusl

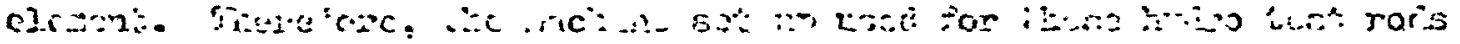

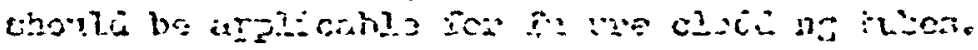

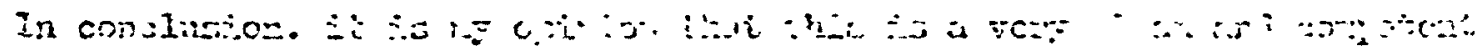

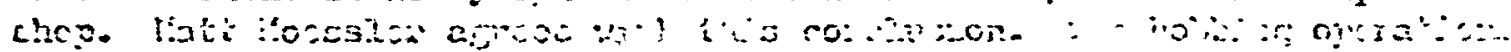

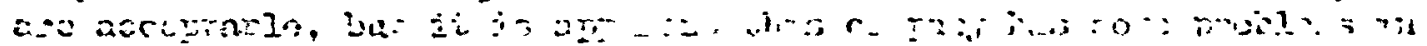

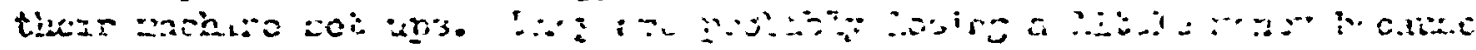


PAGE 19

IF To: D.R. Cariteil

Frow: F.C. 5 cibrag

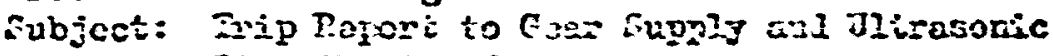
Disiributisg co.
Pase 2

April 30, 1971

of trial ani ciror set-lin of the hebjirz rachine ae oprosed ts calcu-

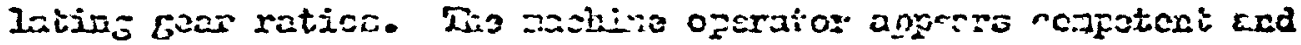

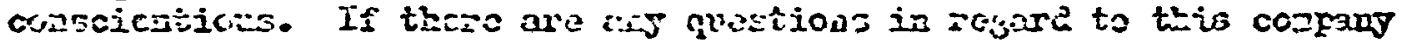

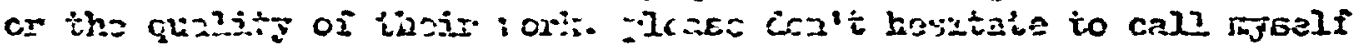
or lieti losisicr.

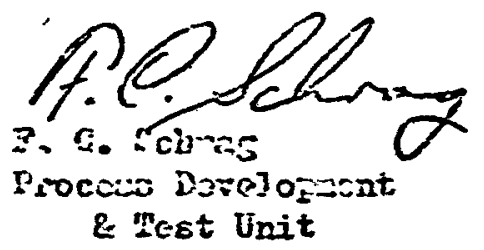

129

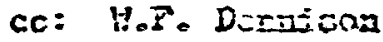

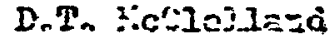

i!. I!oessIn: 